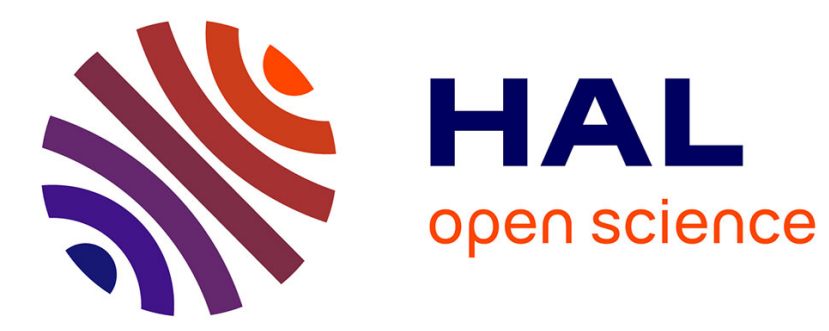

\title{
Triggering of Negative Corona
}

\author{
M. Laan, P. Paris, V. Repän
}

\section{To cite this version:}

M. Laan, P. Paris, V. Repän. Triggering of Negative Corona. Journal de Physique IV Proceedings, 1997, 07 (C4), pp.C4-259-C4-270. 10.1051/jp4:1997421 . jpa-00255577

\section{HAL Id: jpa-00255577 https://hal.science/jpa-00255577}

Submitted on 1 Jan 1997

HAL is a multi-disciplinary open access archive for the deposit and dissemination of scientific research documents, whether they are published or not. The documents may come from teaching and research institutions in France or abroad, or from public or private research centers.
L'archive ouverte pluridisciplinaire HAL, est destinée au dépôt et à la diffusion de documents scientifiques de niveau recherche, publiés ou non, émanant des établissements d'enseignement et de recherche français ou étrangers, des laboratoires publics ou privés. 


\title{
Triggering of Negative Corona
}

\author{
M. Laan, P. Paris and V. Repän
}

University of Tartu, Institute of Experimental Physics and Technology, Tartu Tähe Str. 4, EE2400 Estonia

\begin{abstract}
The parameters of both low-current and high-current modes of DC negative corona discharge are described. The experiments were carried out in point-plane gaps in stagnant media of pure nitrogen, nitrogen, ambient air and in a dust-free air flow. A number of different types of point electrodes were used, including points covered by layers of $\mathrm{TiO}_{2}$ and $\mathrm{HfO}_{2}$ of controlled thickness. An overview of different methods of the triggering of the high-current mode is given. Discharges were initiated using X-ray radiation, laser radiation, UV radiation from different sources, and flux of positive ions. The correspondence of the model of dielectric switching mechanism as the triggering mechanism of high-current mode to the experimental results is discussed. Besides, the possible mechanisms limiting the repetition rate of Trichel pulses at its lower values $\left(<10^{4} \mathrm{~s}^{-1}\right)$ are analysed.
\end{abstract}

\section{INTRODUCTION}

There are two modes of DC negative corona: the low-current (LCM) and the high-current (HCM) ones. These modes are observed in electronegative as well as in non-attaching gases ([1], [2]). Near the onset potential in electronegative gases the HCM exists in a pulsed manner and the parameters of the pulses are determined by the processes in the discharge gap. In non-attaching gases the HCM is controlled by the outer circuit: the discharge may be a steady-state one [1] or relaxation-type oscillations may occur [3]. The processes leading to the oscillations are not treated here; i.e. we assume that the outer circuit ensures the establishment of a steady-state discharge of the HCM.

The study of the triggering of negative corona has a long-term history: the first systematical studies of pulsed corona (Trichel pulses) in air were carried out in a point-plane gap by Loeb et al [4]. Their most important findings were:

- The onset potential of repetitive Trichel pulses is badly reproducible, it has a strong dependence on the point prehistory.

- The onset potential of pulses does not depend on point material.

- The stable triggering of Trichel pulses was achieved when the points where covered by the specks of some insulating material. It was concluded that the Malter effect has a crucial role for the triggering of the pulses.

- Measurements of initiation of Trichel pulses by UV light gave contradictory results. Radiation of higher intensity suppressed the pulses, at lower intensities the pulses were well triggered.

We had two important reasons to carry out a cycle of studies of the triggering of the HCM of negative corona. Firstly, as was shown by experiments [1], during the first tens of nanoseconds of the LCM-HCM transition neither the spatio-temporal development of the light emitted by discharge nor the temporal changes of the current depend on gas composition. So, very likely the mechanisms of the triggering processes are similar. Furthermore, there is a striking similarity between the LCM-HCM transition and the development of instabilities in high pressure bulk discharges in a homogeneous field [5]. For this reason the study of the triggering of the HCM of negative corona might serve as a model of the rise of nearcathode ionisation instabilities in the case of bulk discharges. Secondly, the negative corona proved to be a very sensitive detector of electronegative trace gases: recording the repetition rate of corona pulses as a 
function of the mean current of corona, it is possible to determine ppm-level concentrations of $\mathrm{SF}_{6}$ in argon [6] from the slope of the dependence. Unfortunately the effect of ageing interferes: in a stagnant medium at a certain gas composition the slope of the dependence altered with time [7]; i.e. the probability of the rise of a Trichel pulse changes with time. Whether the effect was caused by the changes of the gas composition or the point emissivity was not clarified.

As the field strength at the cathode surface $\left(\sim 10^{5} \mathrm{~V} / \mathrm{cm}\right)$ is close to that of vacuum breakdown, it was suggested [8] that the triggering mechanisms should not differ much and so the HCM of corona is triggered by the Fowler-Nordheim type burst-like emission of electrons. The studies of two research groups strongly influenced the set-up of this assumption:

- The similarities between the triggering mechanisms of vacuum breakdown and that of high pressure discharges have been emphasised and experimentally studied by Latham et al [9]. According to a detailed model developed (e.g. [10]), the emission starts from the site of the cathode covered by a dielectric inclusion. This 'dielectric switching mechanism' is supported by the measured energy distribution function of emitted electrons.

- In a number of publications Mesyats et al (e.g. [11]) have dealt with the viewpoint of the common nature of the triggering mechanisms of vacuum breakdown and gas discharges. They explain that in a wide range of discharge types (inc. corona) any discharge starts due to the formation of explosive centres 'ectons'.

The purpose of the present paper is to give an overview of the different methods used for the initiation of negative corona, the experimental results, and to discuss the correspondence of the results with the model proposed.

\section{EXPERIMENTAL}

\subsection{Discharge gaps and gas media}

All the experiments were made in point-plane gaps. In most of the cases (if not mentioned otherwise) the gap spacing was $4 \mathrm{~cm}$ and the point electrode was stressed. The point electrodes were hemispherically capped wires of diameter $1 \mathrm{~mm}$. The point electrodes were made of platinum (Pt), aluminium (Al), copper $(\mathrm{Cu})$, molybdenum (Mo) and graphite $(\mathrm{C})$. Their curvature radius differed from $0.5 \mathrm{~mm}$ not more than 10 $\mu \mathrm{m}$. Both previously non-oxidised $\mathrm{Cu}$ and Mo electrodes and the ones oxidised in hot air were used. A number of $\mathrm{Mo}$ and $\mathrm{Cu}$ points were covered by layers of $\mathrm{TiO}_{2}$ and $\mathrm{HfO}_{2}$ of different thickness (1-100 nm) [12]. The morphological changes caused by discharge at the point surface and the microprobe $x$-ray spectra of different sites at the point surface were recorded with the help of scanning electron microscope.

The experiments were made in four different media: stagnant media of pure nitrogen, nitrogen, ambient air, and in a dust-free air flow.

Pure nitrogen: the discharge chamber was evacuated to $5 \cdot 10^{-8}$ Torr and warmed up to $100{ }^{\circ} \mathrm{C}$, the duration of a pumping was at least $10 \mathrm{~h}$. The chamber was filled with pure nitrogen (nitrogen 4.6).

Nitrogen: the discharge chamber was evacuated to $<10^{-5}$ Torr and was filled with the nitrogen of the same purity as in the first medium.

Ambient air: the measurements were made with laboratory air.

Dust-free air flow: before the entering in a discharge chamber the air flow passed a high efficiency fibre filter; only the particles of less than $10 \mathrm{~nm}$ were recorded and their concentration was $<10^{3} 1 / \mathrm{cm}^{3}$.

\subsection{Common regularities of LCM-HCM transition}

A sketch of a typical current pulse corresponding to the transition is presented in figure 1 . Depending on the gas medium, its pressure and point material, a step $i_{S}$ at the leading edge of the current pulse is more or less pronounced. In nitrogen a well-pronounced step is recorded in the case of $C$ point in a wide range of pressures (up to atmospheric) while for Mo point a step is observable only in the limits of 100-150 Torr 
[8]. If the other experimental conditions (pressure, point type etc.) are maintained the same, the leading edge does not depend remarkably on the gas composition used.

In contrast, the decay of a pulse has a strong dependence on medium. At atmospheric pressure in pure nitrogen and also in un-used nitrogen the current peak $i_{\text {max }}$ is followed by a slow decay and after $1.5 \mu$ s a steady-state $\mathrm{HCM}$ is established [1, 13]. After the discharge running some time in nitrogen, the steady HCM is replaced by a pulsed discharge (figure 1). The pulse duration $\tau$ diminishes gradually with the increasing running time of discharge. In ambient air and air flow only a pulse form of the HCM exists and at the atmospheric pressure the typical decay time of the current is $\sim 100 \mathrm{~ns}$.

\subsection{LCM in stagnant media}

The LCM in stagnant media are self-sustained ones, i. e. they exist without external irradiation.

The curve $\mathrm{ABCDEF}$ presented in figure 2 belongs to the discharge in pure nitrogen at near-atmospheric pressure [1]. The part $\mathrm{AB}$ is the $i-U$ characteristic for the LCM. The current of LCM is time-dependent: at a certain voltage the current diminishes with time, the decay-time has the range of tens of minutes. In [2] a similar curve of LCM was recorded in pure nitrogen at higher pressures. At the point $B$ the transition BCDE to the HCM starts. If the protective resistor is small enough, the current pulse corresponding to the transition is very close to the one presented in figure 1. The part EF belongs to the HCM, the current is determined by a protective resistor.

It should be pointed that the point electrode was used for a long time and its surface was covered by craters.

In ambient air [1] the $i-U$ curve of the LCM $\left(\mathrm{A}^{\prime} \mathrm{B}^{\prime}\right.$ in figure 2) is more erratic, the dependence both on the point type and the sample was observed; e.g. for an oxidised Al point the current $>10^{-12}$ A was recorded only at voltages $U$ $>7 \mathrm{kV}$. At the point $\mathrm{B}^{\prime}$ the discharge transits to the HCM; i.e. to the regime of repetitive Trichel pulses. The voltage $U_{0}$ corresponding to $\mathrm{B}^{\prime}$ is the onset potential.

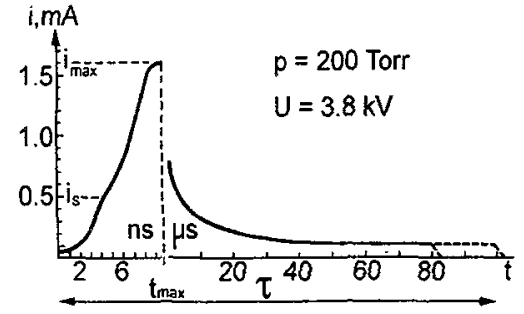

Figure 1: Typical current pulse in nitrogen, Al point; $i_{s}$ - current step; $i_{\max }$ - current peak; $\tau$ - pulse duration.

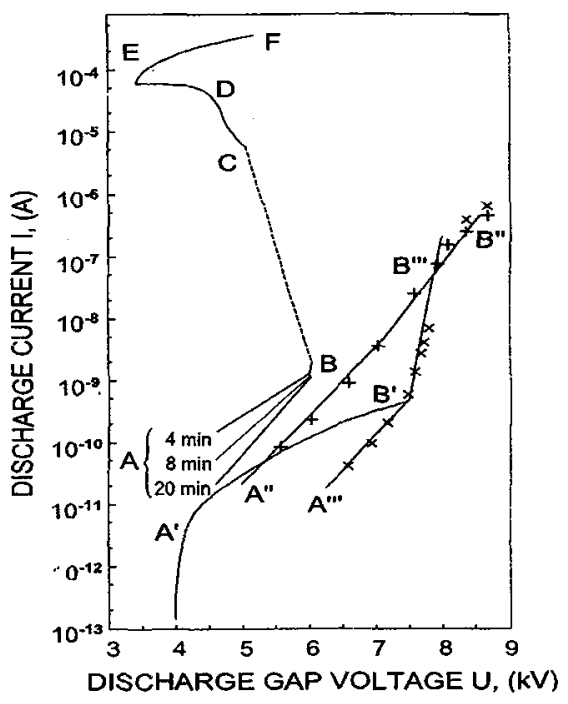

Figure 2: $i-U$ characteristics for negative coronas, $\mathrm{Pt}$ point; $\mathrm{ABCDEF}$ - pure nitrogen, stagnant medium, self-sustained discharge; $A^{\prime} B^{\prime}$ - zmbient air, self-sustained discharge; $A^{\prime \prime} B^{\prime \prime}$, $A^{\prime \prime \prime} B^{\prime \prime \prime}$ - air flow, two different intensities of UV light, non-self-sustained discharge.

\subsection{Dependence of repetition rate of Trichel pulses on point properties}

As it is described in sections 2.2 and 2.3, the LCM-HCM transition as well as the LCM are dependent on the point properties. In [14] the influence of the point properties on the parameters of the HCM (i.e. on the repetition rate and the mean current of Trichel pulses) in ambient air is described. $\mathrm{Cu}$ points oxidised in hot air (thickness of an oxide layer is $>250 \mathrm{~nm}$ ) and those covered by $\mathrm{TiO}_{2}$ layers of $5 \mathrm{~nm}$ and $100 \mathrm{~nm}$ thickness were tested. In the case of thick layers $(250$ and $100 \mathrm{~nm})$ there is a huge difference between the repetition rate (RR) - voltage $(U)$ dependencies for a virgin point and for a point influenced by discharge 
for a longer time. The curves presented in figure 3 belong to one and the same point and are recorded in a similar manner: firstly the voltage is increased by a $0.2 \mathrm{kV}$ step $(8.0 \mathrm{kV} \rightarrow 8.2$ $\mathrm{kV} \rightarrow$ etc.) and then decreased (as $8.5 \mathrm{kV} \rightarrow 8.3 \mathrm{kV} \rightarrow$ ). In the case of a virgin point a memory effect is observed: at every voltage the RR depends on its value at the previous voltage and not on the voltage established. At a certain voltage the $R R$ is time-dependent: it gradually increases with time. This change lasts for several minutes and its duration depends on voltage. After this process the point becomes conditioned and the RR- $U$ dependence is nearly a linear one.

For the points covered by thin layers there are no remarkable differences between linear RR- $U$ dependencies for virgin and used points.

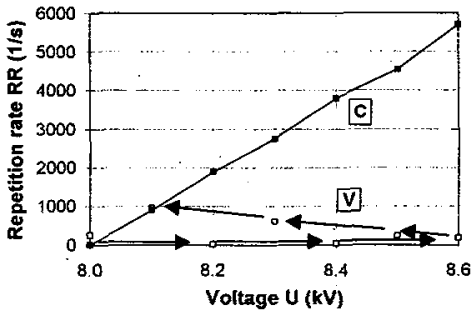

Figure 3: Repetition rate as a function of voltage, oxidised $\mathrm{Cu}$ point; $\mathrm{V}$. dependence for virgin point; $\mathrm{C}$ dependence for conditioned point.

The observations with a scanning electron microscope indicated that due to the surface-discharge interaction a number of micrometer-size craters appeared at the surface of the points covered by thick layers while in the case of thin layers the craters were badly observable or they were missing at all.

The linear dependence of the RR on the mean current of corona seems independent of the point properties.

\subsection{Initiation of LCM-HCM transition in stagnant media}

During the LCM-HCM transition occurring at a certain voltage the current suddenly increases by several orders of magnitude. To understand the nature of these huge changes it is not enough to study only the self-excited (spontaneous) corona pulses. The external triggering gives much more information on the processes leading to the transition.

\subsubsection{X-ray initiation}

In ambient air a flash of x-ray radiation of $5 \mathrm{keV}$ mean quantum energy and of $50 \mathrm{~ns}$ half-width was directed through an aperture in anode to the discharge gap [1]. The repetition rate of flashes was $10 \mathrm{~s}^{-1}$. To minimise the influence of spontaneous pulses the measurements were carried out at voltages close to the onset $U_{0}$. The distributions of delay times of Trichel pulses from the beginning of x-ray pulse were measured for different points. The distribution function varies with the point sample and voltage but always the highest probability of the rise of a Trichel pulse is in the limits $30-40$ ns. When a Pt point is covered by a layer of transformer oil the current of the LCM is practically absent and the onset of spontaneous pulses rises remarkably (up to $1 \mathrm{kV}$ ) compared with $U_{0}$ for non-covered points. Nevertheless the initiated ones start at voltage $U_{0}$. At higher voltages an x-ray flash initiates a series of Trichel pulses.

\subsubsection{Laser initiation}

In nitrogen where the onset $U_{0} \approx 9 \mathrm{kV}$ is considerably higher than in ambient air the corona pulses were triggered using the radiation of a pulsed $\mathrm{XeCl}$ laser $(\lambda=308 \mathrm{~nm}$, pulse duration $\approx 60 \mathrm{~ns})$ [13]. The radiation was directed to the gap through an aperture in the central part of the plane electrode and it was focused near the point surface. The maximum intensity $\sim 10^{7} \mathrm{~W} / \mathrm{cm}^{2}$ is much lower from that needed for the optical breakdown. It is possible to record laser-triggered current pulses starting from the voltage $U$ $\approx 3 \mathrm{kV}$ and starting from $U \approx 7 \mathrm{kV}$ the waveform of a current pulse becomes similar to that of spontaneous pulses in nitrogen (figure 1) with its typical long tail. Decreasing the intensity of radiation by an order of magnitude the time interval between the beginning of a laser pulse and the beginning of current pulse changes from 5 to $90 \mathrm{~ns}$. Very likely the triggering in this case is caused by the heating of the point surface up to the temperatures where the thermionic emission becomes effective. It is proved by the linear dependence of $\ln \left(i_{\max }\right)$ on $\sqrt{U}$ for a number of different radiation intensities. 
The main disadvantage of this way of initiation is that due to the high temperature of the point a large amount of impurities is liberated and the gas composition changes during a short time interval.

\subsubsection{Laser-formed plasma initiation}

Although the initiation of corona pulses by laser radiation described in the previous section is an efficient one, it is hardly possible that such thermal mechanism takes place in the case of spontaneous corona pulses. For that reason another laser-based triggering method was developed [15]. In ambient air the radiation of a pulsed $\mathrm{XeCl}$ excimer laser (pulse of $\approx 50 \mathrm{~mJ}$ energy, repetition rate $10 \mathrm{~s}^{-1}$ ) was focused on the $\mathrm{Pb}$ target at the anode surface. The plasma formed at the target radiates in a wide range of spectra: we recorded a quasicontinuous spectrum in the limits of 200-600 nm. A pulse of plasma radiation has the rise-time of $5 \mathrm{~ns}$ followed by an exponential decay. The pulse duration is $\approx 40 \mathrm{~ns}$. The method used allows to trigger corona pulses both below and above the onset potential $U_{0}$. The minimum voltage where the triggering is achieved depends to some degree on point type. The delay time changes from $30 \mathrm{~ns}$ at the minimum voltage to $10 \mathrm{~ns}$ at the onset $U_{0}$; at higher voltages the delay time remains nearly constant.

Slow but systematic changes of the delay time take place with running time of pulses. Very likely they are caused by the fine dust particles emitted from the target: as detected with an Auger spectrometer there were specks of $\mathrm{Pb}$ at the point surface.

\subsection{Device for the study in dust-free air flow}

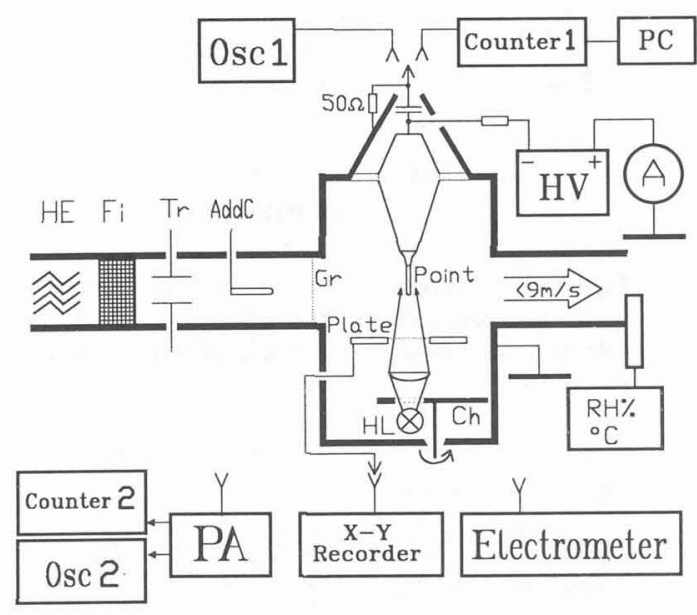

Figure 4: Device for studies in dust-free air flow; Osc1, Ocs2 oscilloscopes; A - ammeter; PC personal computer; HE - heat exchanger; $\mathrm{Fi}$ - fibre filter; $\mathrm{Tr}$ - ion trap; AddC - source of additional corona; $\mathrm{Gr}$ - calibrated grids; HL - hydrogen lamp; $\mathrm{Ch}$ - chopper; $\mathrm{RH} \%,{ }^{0} \mathrm{C}$ - hygrometer; PA - preamplifier.
During experiments in a stagnant medium a number of accumulation processes may occur: fine dust particles created by discharge accumulate; gas composition changes due to plasma-chemical reactions; the heating of gas takes place. All these factors may influence the ionisation processes in gas as well as the emissivity of the cathode. Experiments in moving media give a possibility to diminish the number of uncontrolled parameters. A detailed description of the device presented in figure 4 is given in [12] and [16].

The rate of the air flow was changed from 10 to $45 \mathrm{l} / \mathrm{s}$. With the help of a heat exchanger HE it was possible to fix the temperature in the limits 15 $30^{\circ} \mathrm{C}$. The dust and ions in the entering ambient air were removed by the filter Fi and the ion trap $\mathrm{Tr}$, respectively. The temperature, relative humidity and pressure were recorded.

It was possible to direct a flux of positive ions into the discharge chamber using a source of additional positive corona AddC. Depending on the point shape of AddC the ion flux was a steady state or pulsed $\left(>10^{4} 1 / \mathrm{s}\right)$ one. The flux was changed by the grids $\mathrm{Gr}$. The mean current of ions was recorded with the ammeter $A$.

To radiate the point with UV light $(\lambda>200 \mathrm{~nm})$ a hydrogen lamp HL $(20 \mathrm{~W})$ was used. The intensity of light was changed by calibrated slits. For temporal studies a chopper $\mathrm{Ch}$ with variable apertures was used.

A high voltage supply $\mathrm{HV}$ allowed to change the voltage with the smallest step of $10 \mathrm{~V}$. Mean currents were measured with the ammeter $A$ and an electrometer. The waveforms of Trichel pulses were recorded with an oscilloscope Osc1 and the repetition rates with a counter 1 and a personal computer PC. The counter 1 was also used for the measurements of time intervals between light pulses and the Trichel 
ones. Slow $(>1 \mathrm{~s})$ temporal changes were recorded by an $\mathrm{X}-\mathrm{Y}$ recorder. For the detection of faster processes of the LCM a preamplifier PA, an oscilloscope Osc2 and a counter 2 were used.

\subsection{LCM in air flow}

If the gap is not influenced externally, in an air flow at voltages less than $U_{0}$ (onset in a stagnant air) a self-sustained LCM is missing $\left(i<10^{-12} \mathrm{~A}\right.$ ). The discharge current appears only when the $U V$ radiation is directed to the point. At the same gas density, voltage and the intensity of the UV light the current recorded depends on the prehistory of the point: for how long the air flow has been switched on; whether before the recording the point has been illuminated and whether the discharge has been running. At an applied voltage $U<U_{0}$, after switching-on the air flow and the light the current increases gradually and it takes tens of minutes before a more or less stable current value is established.

\subsubsection{Static characteristics}

For the reasons described above, before the recording of the mean current $i$ of the LCM as a function of voltage $U$ every point passed a conditioning procedure described in [12]. After the conditioning a good day-to-day as well as sample-tosample (for a certain point type) reproducibility was achieved. In figure 5 (for $\mathrm{Pt}$ point see also figure 2) a set of $i-U$ curves registered at a constant light intensity $I$ is presented. The recording of a curve was stopped at the voltage where the first Trichel pulses appeared. The $i-U$ characteristics for different point types have the same typical parts: at lower voltages the slope of the dependence is nearly constant and does not depend on point type; at higher voltages an upcurving of the dependence takes place. At every voltage the current has a strong dependence on the insulating film which covers the point surface as well as on its thickness; the maximum difference of currents is more than three orders of magnitude. The current is determined by the insulator layer and not by the substrate material; in figure 6 the $i-U$ curves

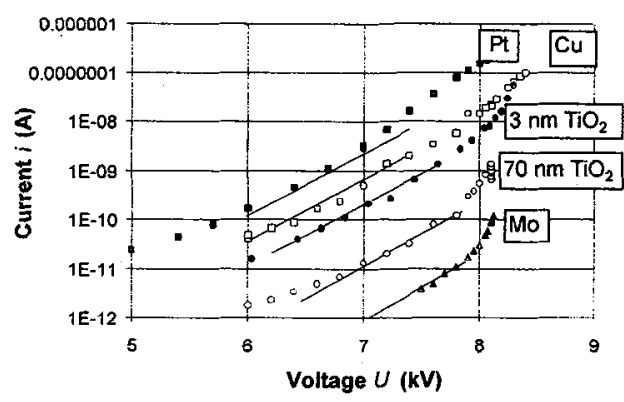

Figure 5: $i-U$ curves of $\mathrm{LCM}$; $\mathrm{TiO}_{2}$ layers are on Mo substrate; $\mathrm{Cu}$ point is a previously oxidised one.

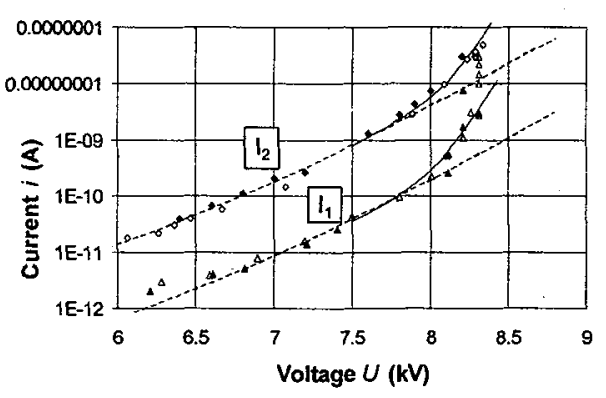

Figure 6: $i-U$ curves for points covered by $3 \mathrm{~nm}$ layer of $\mathrm{TiO}_{2}$; open and filled symbols - $\mathrm{Cu}$ and $\mathrm{Mo}$ substrate, respectively; relationship of light intensities $I_{2} / I_{I} \approx 50$.

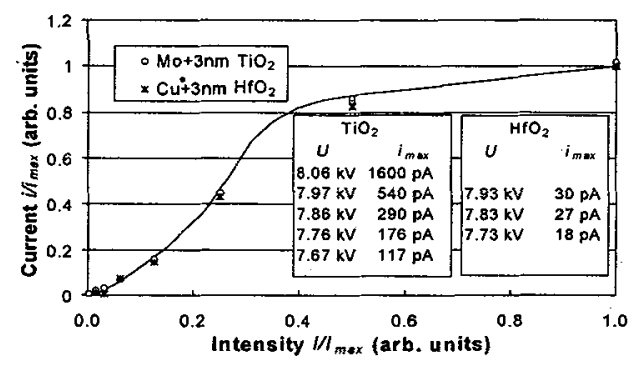

Figure 7: normalised current of LCM as a function of intensity for two point types; $i_{\max }$ corresponds to the maximum intensity used.

for two different substrate material coincide. Another finding follows from this figure: at a certain voltage the relation of currents $i_{2} / i_{1}$ is not equal to that of light intensities $I_{2} / I_{1} \approx 50$. At every voltage the current $i$ is a non-linear function of intensity $I$, as is demonstrated by the curve in figure 7 . It should be emphasised that the dependence is nearly the same for all the curves although the difference between $i_{\max }$ is up to two orders of magnitude. 


\subsubsection{Temporal dependencies}

The results presented in this section are our very recent ones [16]. The temporal changes of the LCM were recorded using a preamplifier of $1 \mathrm{MHz}$ bandwidth. The noise level allowed to record signals starting from $5 \mathrm{nA}$. A chopper $\mathrm{Ch}$ was used to form light pulses (pulse duration of $1.8 \mathrm{~ms}$, rise-time of 50 $\mu$ s and repetition rate of $50 \mathrm{~s}^{-1}$ ). Typical recordings of the current of LCM are presented in figure 8: on a steady level of a current pulse (which duration equals to that of the initiating light pulse) there is a number of sharp current spikes of varying amplitude. The noise level allowed to record spikes not more than $150 \mathrm{~V}$ below the voltage where the Trichel pulses started.

The distribution of spikes of different amplitudes during a light pulse is non-random; overlapping a number of recordings presented in figure $8 \mathrm{~A}$ a certain regularity appear: spikes of larger amplitudes start preferably at the initial part of the light pulse. The probability of the rise of another large spike during the same light pulse is very low.

The amplitude distributions of the spikes for Pt point were measured as functions of voltage and light intensity. At a constant intensity the increasing of the voltage by $150 \mathrm{~V}$ causes a 10-fold rise in the number of spikes whose peaks exceed a certain value while no changes of the distribution function (figure 9) was found.. At a fixed voltage the light intensity influences both the steady level of the current and the total number $N$ of spikes. The lowering of the intensity from its maximum value to $1 / 64$ of it reduces the steady component from tens of nanoampers to a nondetectable level. The dependence of the total number of spikes $N$ on intensity is a non-linear one having a trend of saturation. The amplitude distribution of spikes remains unchanged.

The number of spikes depends strongly on temperature: its alteration by only a few degrees causes remarkable changes in the number of spikes. At the temperatures $<30{ }^{\circ} \mathrm{C}$ and the $\mathrm{RH}<40 \%$ there are no spikes between light pulses. At higher humidities the number of spontaneous spikes increases drastically.

The spikes recorded were integrated ones. To estimate the actual waveform of them a faster but less sensitive preamplifier was used: the rise-time of spikes is less than $10 \mathrm{~ns}$ and the halfwidth of a pulse has a. $100 \mathrm{~ns}$ order of magnitude. Only the largest spikes were recorded this way and their peak value is $\approx 10^{-6}$ A. No changes of the halfwidth of the spikes were noticed.

\subsection{Triggering of Trichel pulses in an air flow}

As a rule in an air flow the spontaneous Trichel pulses do not start at voltage $U_{0} \approx 8 \mathrm{kV}$ corresponding to the onset in stagnant air; for that considerably higher voltages (up to $9 \mathrm{kV}$ ) are needed. The voltage where the spontaneous pulses start is determined by the point sample: the less dust specks and/or protrusions are observed at the point surface the higher the voltage is. For external triggering experiments the samples having the highest mentioned voltage were selected from every point type.

\subsubsection{Triggering by an ion flux}

'Dielectric switching mechanism' [10] as well as Malter-like emission [4] assume the presence of insulating inclusions at the cathode. Indeed, as it was proved in our studies even in the case of previously 
non-covered Pt point Trichel pulses start from a site where insulating inclusions are present. So there are sites at the point where the accumulation of the charge is possible and it may trigger Trichel pulses. To test it, a flux of externally created positive ions was directed to the point; the experimental procedure is described in [12]. The triggering becomes possible already at voltages below $U_{0}$. A set of dependencies typical for every point type is presented in figure 10. It is remarkable that there is an onset flux of ions starting from which the triggering becomes possible. Unlike the $i-U$ characteristics of the LCM it was not possible for the RR to fix clearly a dependence on point type.

Using triggering by a pulsed flux of ions the dependencies of the RR on the mean current of ions were similar to those in figure 10 . The only difference is that the fluctuations of the RR are somewhat larger than those for a steady flux.

\subsubsection{Triggering by steady radiation of integral UV light}

A hydrogen lamp like that in section 2.7 was used. A very stable triggering was achieved starting from very low values of the RR as it is demonstrated in figure 11 . Already a very faint radiation (e.g. light scattered from the walls of the chamber) triggers Trichel pulses. Higher intensities of radiation suppress Trichel pulses. The latter effect depends on the point type and it is especially remarkable for the $\mathrm{Pt}$ point (figure 2, curves $\mathrm{A}^{\prime \prime} \mathrm{B}^{\prime \prime}$, $\left.A^{\prime \prime} B^{\prime \prime \prime}\right)$ : 50 times higher intensity increases the starting potential of the initiated Trichel pulses by $\approx 500 \mathrm{~V}$.

The RR as a function of intensity is presented in figure 12: the point used did not pass the preliminary training procedure (section 2.7.1.) and so its current of the LCM is small compared with that achieved after training; the RR saturates already at low intensities of light.

\subsubsection{Triggering by monochromatic light}

In these experiments the gap spacing was $1.3 \mathrm{~cm}$. For triggering the light from a Xe lamp $(1 \mathrm{~kW})$ which passed a monochromator [16] or that of an excimer source $(\lambda=308$ nm) [17] was used.

The spectral intensity at the point plane as a function of wavelength (figure 13) was determined by measuring the spectral power and estimating the radiated area.

The RR depends on wavelength (figure 13) and the dependence is different for different point types. The dependencies of the $R R$ on intensity recorded for two different wavelengths (figure 14) have the same trend of saturation as in the case of the measurements with integral light (figure 12). The difference between the values of the

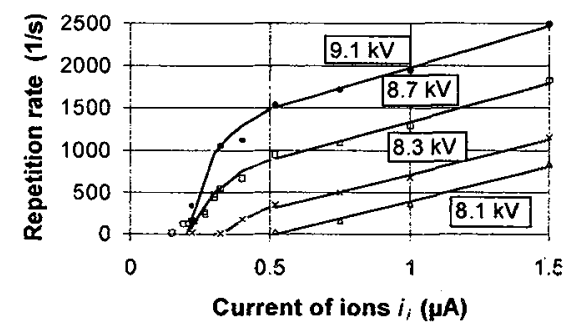

Figure 10: RR as a function of current of ions $i_{i}$ : previously oxidised $\mathrm{Cu}$ point.

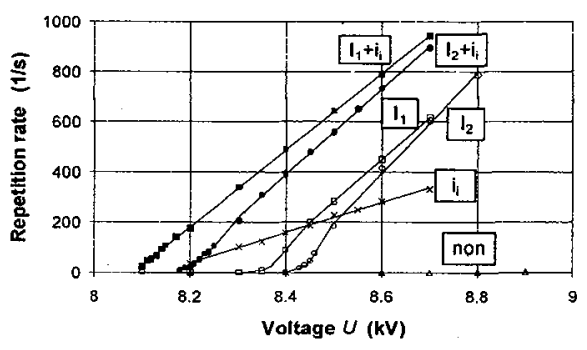

Figure 11: RR as a function of voltage; triggering both by UV light and flux of ions; relationship of intensities of UV light $I_{2} / I_{1} \approx 50$; current of ions $i_{1}=$ $0.77 \mu \mathrm{A}$; non $-\mathrm{RR}$ of spontaneous pulses.

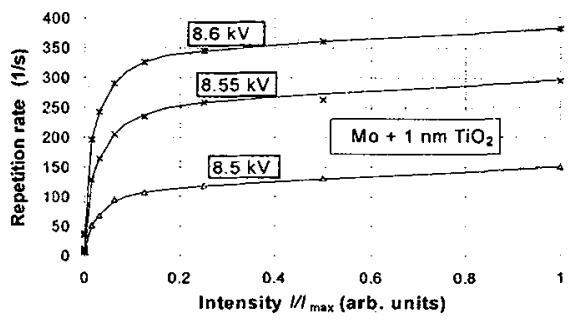

Figure 12: RR as a function of intensity; the point is not conditioned.

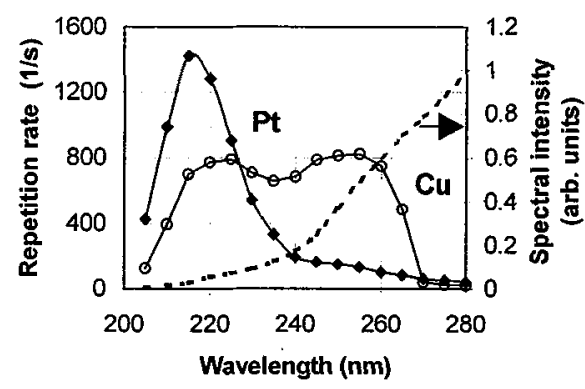

Figure 13: RR as a function of wavelength; gap spacing - $1.3 \mathrm{~cm}$; dashed line- intensity at the point surface. 
RR where the saturation is achieved is remarkable. Furthermore, for the Trichel pulses initiated using an excimer source $[17]$ the saturation occurs at the $R R \sim 10^{4}$ $\mathrm{s}^{-1}$.

Again (see section 2.7.2.) the temperature changes had a huge influence on the discharge parameters. Temperature seems to be an independent parameter, as concluded from reducing the experimental results to the same gas density. The influence of temperature are demonstrated also by experiments with infrared $(\lambda=1-3 \mu \mathrm{m})$ radiation of a 50 $W$ halogen lamp [17]. In an air flow of the rate $20 \mathrm{l} / \mathrm{s}$ and temperature of $28{ }^{\circ} \mathrm{C}$ the radiation of excimer lamp initiates $10^{3}$ Trichel pulses per second. During a $70 \mathrm{~s}$ action of infrared radiation the RR increases to $>5000 \mathrm{~s}^{-1}$. In the mentioned experimental conditions radiation warms up only the point surface and maybe a very thin gas layer near it.

\subsubsection{Temporal characteristics of triggering}

Trichel pulses were triggered by light pulses similar to those described in section 2.7.2. The limits of voltage were chosen so that the number of Trichel pulses created during a light pulse was close to one [16]. At a certain voltage the delay time (the time interval between the beginning of the light pulse and beginning of a Trichel pulse) as a function of light intensity was recorded. Delay time distribution (figure 15) becomes narrower with the increasing intensity. The same trend takes place while recording the distribution as a function of voltage at a constant light intensity.

Both formative and statistical time lags were determined from Laue plots. In the limits of the intensities and voltages used the formative time lags are $<50 \mu$ s; i.e. close to the rise-time of the light pulse. Statistical time lags determined from the initial linear parts of Laue curves in figure 16 for light intensities $I, I / 4, I / 8$ are $82 \mu \mathrm{s}, 149 \mu \mathrm{s}$ and $350 \mu \mathrm{s}$, respectively.

\section{DISCUSSION}

\subsection{Triggering mechanism}

As it is modelled by Morrow [18], at lower pressures only few electrons are needed for triggering and an avalanche series supported by a cathode feedback $\gamma$ mechanism leads to the rise of a Trichel pulse. At atmospheric pressures the onset potential is independent on point material. This effect may be caused by gas layers absorbed at the point surface thus making the actual $\gamma$ insensitive to the point material [4]. In contrast to this assumption, in an air flow the current of the LCM caused by UV light is very sensitive to the cathode type (figure 5). In spite of this the voltages starting from which the triggering becomes

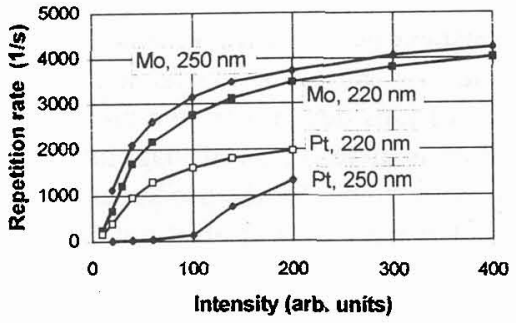

Figure 14: RR as a function of UV intensity for two different wavelength; $U=6.9 \mathrm{kV}$.

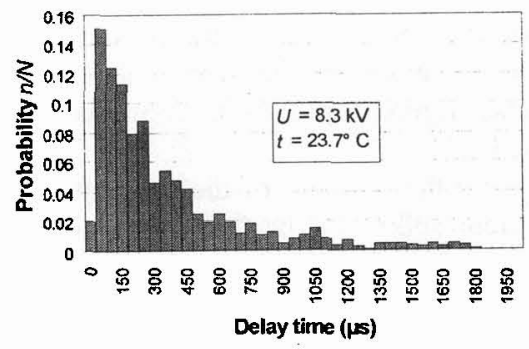

Figure 15: Delay time distribution of Trichel pulses.

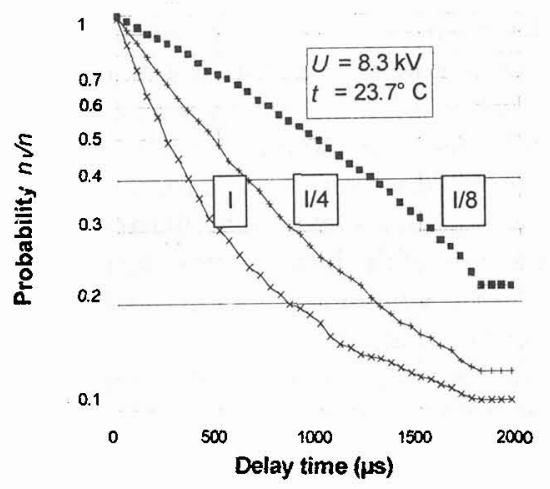

Figure 16: Laue curves of delay time distribution of Trichel pulses for three intensities of UV light. 
possible for different point types lie within narrow limits $(\approx 200 \mathrm{~V}$, figure 5$)$. So it is unlikely that the avalanche series of $\gamma_{p h}$ feedback lead to the rise of Trichel pulses. The other possibility that the avalanche series are caused by ion feedback $\gamma_{i}$ is invalid as well: the number of Trichel pulses created by a pulsed flux of ions depends on the mean value of the current of ions (section 2.8.1.) and not on the peak value which is three orders of magnitude higher. Consequently, the ordinary cathode feedback mechanisms are not responsible for the processes leading to the triggering of corona pulses.

For triggering at atmospheric pressures it is not enough to liberate few electrons from the cathode: although during an initiating light pulse (section 2.7.2.) there is always a steady component of the current, the temporal distribution of the probability of the rise of Trichel pulses (figure 15) corresponds to the temporal distribution of the current spikes of the LCM having large amplitudes (figure 8A). So it is natural to suppose that a Trichel pulse is triggered by a larger current spike. A correspondence between the time intervals between larger current spikes (figure $8 \mathrm{~B}$ ) and the statistical time lags of Trichel pulses (section 2.8.4.) supports this assumption. The spikes recorded are not the fluctuations of the photoassisted current caused by the avalanche statistics; already the non-random distribution of spikes during a light pulse (figure 8) proves it. Consequently, the 'particles' which trigger Trichel pulses are current spikes of the LCM.

So the problem of the triggering of negative corona is reduced to the mechanism of the rise of the current spikes of the LCM. It is likely that spikes are caused by the switch-on of an extra emission mechanism. As a Trichel pulse always arises from a site where an insulator inclusion is present [12], let us analyse the nature of the initial currents from the viewpoint of a metal-insulator-gas (MIG) system like it is done for metal-insulator-vacuum systems [10].

A sketch in figure 17 corresponds to the situation where an emissive site is switched on; i.e. both traps and surface states are filled, the metal-insulator barrier is thin enough for tunnelling, and the barrier of the insulator-gas interface is lowered to the level which allows the emission of thermoelectrons [10]. As in our conditions the current is not recorded without UV radiation (section 2.7.), the voltage drop across the MIG system is too low for stable tunnelling. Another difference from the sketch presented is that in our case the layers of $\mathrm{TiO}_{2}$ and $\mathrm{HfO}_{2}$ were very thin ones and so the linear parts at the sketch belonging to the bulk of the insulator may be absent.

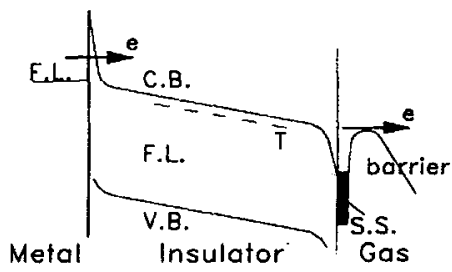

Figure 17: Configuration of energy gaps; F.L. - Fermi level; C.B. - conduction band; V.B. - valence band; T - traps; S.S. surface states.

So without external assistance both the surface states and traps are empty and the emission current is absent. Under the influence of UV radiation the electrons are transferred from the valence band to the conduction band. These electrons may be emitted thermionically (thus causing the steady component of the LCM current) or captured by empty traps and surface states. The higher the UV intensity is the more surface states are filled. The electrons filling the surface states will screen the bulk of the insulator from the applied field. This is a probable reason for a non-linear dependence of the mean current of the LCM on UV intensity (figure 7). UV radiation not only causes the emission but also changes the band configuration. Due to the UV action the traps and surface states are more-or-less filled the increasing of the applied voltage makes the tunnelling possible and a burst of electrons is injected to the insulator. A part of them is thermionically emitted, another part is captured by surface states, thus interrupting the emission. Of course the similar burst-like emission may be caused not only by UV light but also by ions accumulating at the insulator and thus increasing the voltage drop over the MIG system (section 2.8.1.) or by the heating of the point due to the laser radiation (section 2.5.2.).

The burst of electrons emitted is amplified due to gas amplification and is recorded as a current spike. The higher the voltage is the larger the spikes are and the shorter is the time interval between spikes. The experimental findings described in this paper (including long-term changes of emissivity during the conditioning) are qualitatively explainable on the basis of the model proposed.

Besides, some speculations on the influence of the gas composition on the triggering characteristics are possible. Depending on the gas absorbed at the electrode it may cause upward as well as downward 
bending of energy zones near the insulator-gas interface, thus changing the efficiency of emission. Oxygen and water vapour are bending the zones downward; maybe that is a reason why in humid air spontaneous spikes (and so Trichel pulses) arise more frequently (section 2.7.2.) and the onset of negative corona in nitrogen (section 2.5.2.) is considerably higher than in air.

\subsection{Repetition rate}

At a certain voltage and under conditions where spontaneous corona pulses are practically missing the $R R$ is controlled by a flux of ions and/or by some kind of radiation (e.g. figures 10,12). Whether the control of these triggers is caused by their action to the gas or to the cathode is not obvious.

The radiation may cause the detachment of electrons from negative ions existing in the gap, thus diminishing the influence of negative space charge. As a result Trichel pulses may arise more frequently. The same effect may be caused by a flux of positive ions - the concentration of negative ions may diminish due to the ion-ion recombination. The experiments with monochromatic light contradict the described explanations: the dependencies in figure 13 give the basis to confirm firmly that the repetition rate is determined by the emission of the point as there is a strong dependence of the RR on the point type.

The RR as a function of the intensity of initiating particles/radiation has always a trend of saturation. First of all the limitation of the rise of the RR may be caused by the space charge of negative ions created by the Trichel pulses. Calculations based on literature data for field distribution [19] and the mobility of negative ions [20] give drift times $T_{d}=(2.2-2.6) \mathrm{ms}$ for $4 \mathrm{~cm}$ gap and voltages used (figures 10,11, 12) and $T_{d} \approx 0.25 \mathrm{~ms}$ for $1.3 \mathrm{~cm}$ gap (figure 14). It means that starting from $\mathrm{RR}>1 / T_{d}$ the negative space charge created by Trichel pulses accumulates in the gap thus reducing the field (and also field-dependent emission) at the point surface. Indeed, the saturation of the dependencies recorded starts at the values of the RR close to the calculated.

For $4 \mathrm{~cm}$ gap at close voltages the level of saturation of the RR differs for different point types 10 times (figures 10,12); so there must be another reason for the creation of limiting space charge: it may be created by the steady component of the current existing between Trichel pulses. This steady component should be different for different point types like in the case of the LCM (figure 5) and for this reason the value of the $R R$ at which the saturation is achieved is different for different point types. The limitation of the RR by the steady component is also the probable reason.why higher intensities of UV light prevent the rise of larger current spikes and so at higher UV intensities Trichel pulses start at higher voltages (figure 2).

A number of different methods were used for the triggering of negative corona. Once triggered a corona pulse becomes independent on the way of its creation; i.e. its parameters are determined by the gas composition. For this reason in a gas flow where the discharge does not change the gas composition, a linear dependence of the RR on the mean current of corona has the same slope in spite of the fact that spontaneous or in some way initiated pulses are recorded. This statement based on a number of experiments in a dust-free air flow. So the slope of the RR-mean current dependence in a gas flow may be used as an indicator of the gas composition.

\section{CONCLUSIONS}

- Study in a dust-free air flow allows to record the parameters of negative corona with a good reproducibility.

- Even at atmospheric pressures in air the current of the LCM is very sensitive to the surface properties.

- Negative corona is triggered by a short-duration burst-like emission of electrons.

- The dielectric switching mechanism explains a number of experimental findings without any contradictions.

- The common nature of the processes leading to the breakdown both in vacuum and in gases have found its further verification. 
- Study of current spikes of the low current mode is an easy way to collect information about the processes preceding the switch-on of emissive sites.

\section{Acknowledgements}

A common analysis of the processes which determine the repetition rate of Trichel pulses carried out by R.S. Sigmond and especially his contra-arguments to our different interpretations essentially helped us. The studies were supported by Estonian Scientific Foundation (grants No 88 and No 1328).

\section{References}

[1] Korge H., Laan M., Paris P., J. Phys. D: Appl. Phys. 26 (1993) 231-236.

[2] Hernandez-Avila J.L., Nur M., Bonifaci N., Denat A., "D.C. Negative corona discharge characteristics in high density nitrogen in point-plane configuration", XXII ICPIG Hoboken, N.J. USA 31.7-5.8.1995, Contr. Papers 2 pp. 129-130.

[3] Sigmond R.S., "Corona physics and diagnostics", XXII ICPIG, Hoboken N.J. USA 31.7-5.8.1995, Invited Papers pp. 332-344.

[4] Loeb L.B., Electrical coronas (Berkeley \& Los Angeles 1965), ch. 4.

[5] Laan M., Paris P., "Formation of corona pulses", $9^{\text {th }}$ Symp. on El. Processes and Chemical Reactions in Low Temperature Plasma, Small Carpathy, Slovakia 1992, Invited Lectures pp. 201-216.

[6] Sigmond R.S., Goldman A., Goldman M., Laan M., Bjones B., Olsen I.-H., "Detection of electronegative trace gases by corona discharges", $11^{\text {th }}$ Int. Symp. on Plasma Chemistry Loughborough, UK 1993, 4, pp.1315-1320.

[7] Bjones B., Olsen I.-H., Corona discharges in air and argon with added trace gases, Diploma thesis (Trondheim, Norwegian Institute of Technology, Norway 1992), $179 \mathrm{p}$.

[8] Laan M., Perelygin V., "The dependence of negative corona on electrode surface properties", XX ICPIG Pisa, Italy 1991, Contr. Papers 4, pp. 929-930.

[9] Latham R.V., Bayliss K.H., Cox B.M., J. Phys. D: Appl. Phys. 19 (1986) 219-231.

[10] Bayliss K.H., Latham R.V., Proc. R. Soc. Lond. A 403 (1986) 285-311.

[11] Mesyats G. A., Ectons (UIF "Nauka", Yekaterinburg 1993) vol. 1, 2 (in Russian).

[12] Laan M., Repän V., Aarik J., Sammelselg V., J. Phys. D. App:. Phys. (1997), submitted.

[13] Laan M., Paris P., Perelygin V., Acta phys. Slov. 42 (1992) No2, 91-97

[14] Laan M., Repän V., Roos H., "Repetition rate of Trichel pulses and conditioning", $10^{\text {th }}$ Symp. on El. Processes and Chemical Reactions in Low Temperaiure Plasma, Slovakia, High Tatras, 1994, Acta

Physica Univ. Comenianae 35 175-179.

[15] Laan M., Paris P., Repän V., Uustare T., "Laser triggered negative corona", $12^{\text {th }}$ ESCAMPIG European Sect. Conf. on the Atomic and Molecular Physics of Ionized Gases (Noordwijkerhout, The Netherlands 1994), Inv. Lectures and Contr. Papers pp. 358-359.

[16] Laan M., Paris P., Repän V., to be published.

[17] Paris P., Laan M., Tiirik A., "UV light controlled repetition rate of Trichel pulses", XXIII ICPIG, Toulouse, France 1997 Contrib. Papers.

[18]. Morrow R., Phys. Rew. A, 32 (1985) 1799-1809.

[19] Coelho R., Debeau J. J. Phys. D: Appl. Phys. 4 (1971) 1266-1280

[20] Badaloni S., Gallimberti I. Basic data of air discharges (Italy, 1972) Report Upee-72/05, 91 p. 